\title{
Biometrics Identification based Face Image Authentication
}

\section{Thamir Abdul Hafidh Israa Mohamed Khidher \\ Department of Computer Science / Collage of Education Mosul university}

\author{
Received \\ 08 / 02 / 2008
}

\section{Accepted}

$10 / 09 / 2008$

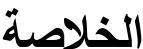

لاقت تكنولوجيا المقاييس الثخصية اهتماماً كبيراً في السنوات الحالية ويعد تمييز الوجه

بصورة اوتوماتيكية احد احدث مجالات تكنولوجيا المقاييس الثخصية. تطورمجال بحث تمييز

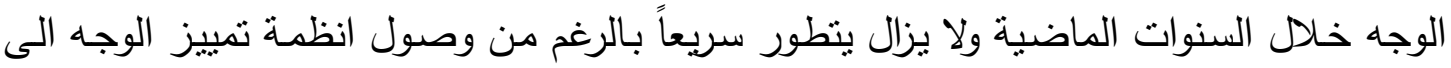

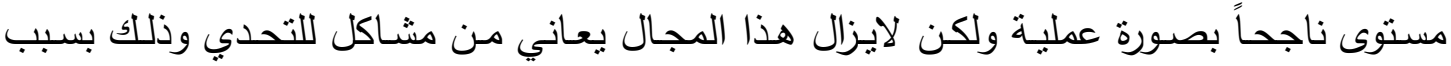

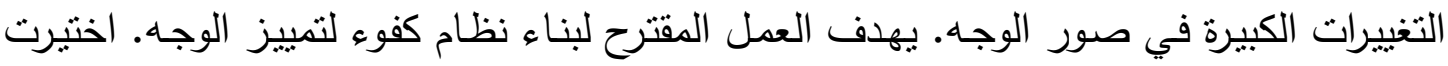

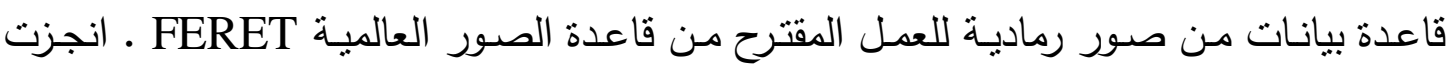

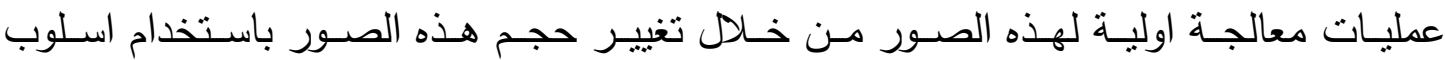
Bilinear اعتمدت Wavelets Transformation لتمبيز الوجه في هذه التجربة وذلك لقوة

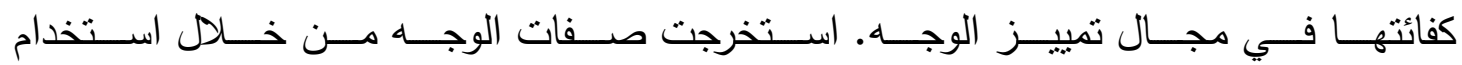

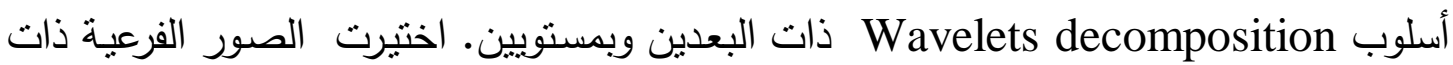
التزددات الافقية والعمودية ذات البعدين. اختيرت هذه الصور وذللك لقلة تحسسها لتغيرات الصورة

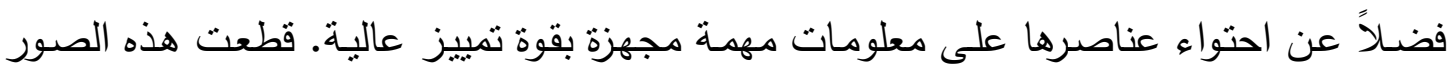
بشكل Blocks واستخدمت العزوم الاحصائية لاستخراج الصفات لكل Block. استخدم العصل الكقترح تقنيات دقيقة لتحليل التمييز والتي عكست نتائج محسنة. هذه النتائج تمنتلة بمقاييس دقيقة

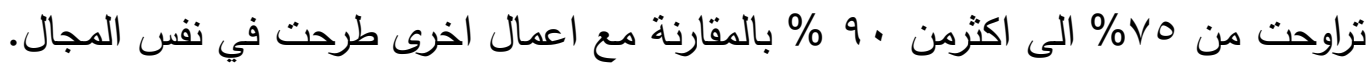

\section{Abstract}

In recent year biometric technology has received a great attention. One of the newest area in biometric technologies is the automatic face recognition. Face recognition has developed over last decades and still a rapidly growing research area. Although, face recognition systems have 
reached a level of practical success but still remains a challenging problem due to large variation in face images. The aim of the proposed work is to build an efficient automatic face recognition. Data base of gray-level images for the proposed system are selected from the Face Recognition Technology FERET. Then primary processing to these images are performed through the downsampled to each face by bilinear method. Then these images were masked by a rectangle that include face region only.

Wavelets transformation is based for face recognition in this experiment due to their powerful efficiency in face recognition area. Face features were extracted through the use of the 2D 2-level wavelets decomposition. The 2D Vertical and Horizontal subimages are selected. These subimages are selected due to their less sensitivity to image variations. As well as their components form the most informative subimage equipped with the highest discriminating power. Then the images are segmented blocks, the Statistical moment is used to extract features per block. The proposed work used accurate techniques to analysis the recognition which reflects significant enhancement results. These results are represented by accurate measures varied from $75 \%$ to more than $100 \%$ compared with other system on the same area.

\section{1- Introduction}

Biometric identification is the technique of automatically identifying or verifying an individual by a physical characteristic or personal attribute [8]. The term "automatically" means that biometric identification system must identify or verify a human characteristic quickly with no interference from the user. Biometric characteristics are divided into behavioral or physical categories. Behavioral biometrics regards such behaviors as signature and typing rhythms. Physical biometric systems use the eye, finger, hand, voice, and face, for identification. A face recognition system would identify person by simply recognize one another by unique facial characteristics [8].

Face authentication recently has received a great attention. It plays an important rule in civilian and military applications [4]. Face authentication classified to two methods they are identification (recognition) and verification [6]. Identification (who am I). Deals with system with input to the system are unknown face and the system responds back with a determined identity from database. Whereas in Verification (Am I Whom I am?) the system accept or reject the query face [4] [6].

Face recognition classified mainly to two methods, they are holistic and geometric method. Holistic method is commonly used in 1990's with well-known approaches Eigenfaces. While, geometric approach become very popular in 1980's, where the distance between eyes and shapes of 
line connecting facial features were used [1][5]. The drawback of this methods is that there performance is not improved because they need threshold which affected by noise images [7].

Various techniques have been proposed in the literature for face feature extraction. These techniques could be classified into four main groups. They are the geometry-based, template-based, color-based and the appearance-based approach. The geometry based approach regards the geometric information as facial features. This information is relative positions and size of the face component such as mouth, noise and eyes. The template-based approach compare and match between the query face and priory defined patterns. Color segmentation, this technique isolate eyes and/or mouth as non-skin color region while regards other color skin as face color region. Finally, appearance-based approaches regard the extracted characteristics from face image as feature and should be included in feature vectors. These approaches are extremely used for face authentification [7].

There exists a various related study in the area of face recognition. Turk and Pentland (1991) use the Principle Component Analysis (PCA) for feature extraction and, nearest neighbor classifier for recognition. Another work by Lee (1996) use 2D Gabor filter for face feature extraction and Dynamic link Architecture for classifier. Also Eickeler, S. et al. (2000) apply 2D DCT for feature extraction and Hidden Markov Model (HMM) as classifier. Indeed, Lawrence et al. (1997) have been use Artificial Neural Networks [11]. These studies have drawbacks the features derived by PCA is variant to illumination causing rapid blur [6] while, Gabor Wavelets are sensitive to illumination change [11]. The proposed work uses appearance-based approaches through gray-level face images. These images are resized to a common size and 2-level wavelets decomposition transform is applied to these images. Only the vertical and horizontal packets are selected for feature extraction process. Then these packets are segmented to blocks. In order to extract feature for efficient face recognition we combine the methods of statistical moments with the direct use of wavelets components. Our proposed work differ from others work in that they use pixel-wise operation but the proposed work use block-wise approach to segment the vertical and horizontal subimages. The waveletface by Chien and $\mathrm{Wu}$ (2002) decompose the image into four packet and construct further subimages [6]. Also they used the eyes, mouth and noise as Region of Interest (ROI) with wavelets [10] while our system uses the whole face in block-wise form.

\section{2- Introduction to Wavelets}

Wavelets are functions that are widely used in image and signal processing. They represent data or other functions from mathematics. Wavelets are similar to Fourier transform in representing function similar 
to sine and cosine, but differ in representing data. Data are represented in both spatial and frequency domain simultaneously [5]. The continuous wavelets transform of $1 \mathrm{D}$ signal $\mathrm{f}(\mathrm{x})$ is computed by using equation (1):

Wf, a $(\mathrm{u}, \xi)=\frac{1}{\sqrt{a}} \int_{-\infty}^{\infty} f(t) h *\left(\frac{t-\xi}{a}\right) d t$

Where $\boldsymbol{a}$ is scaling factor, $\xi$ is the translation parameter corresponds to the location of window, and $\mathrm{h}(\mathrm{t})$ is the transforming function and also called mother wavelet [5] [6] [3]. Discretized operation by re-constraining a and $\xi$ to discrete form. 2D is usually evaluated by using a product of 1D filter [3]. The image is filtered simultaneously in row-wise to high and low pass filter denoted by $\mathrm{H}, \mathrm{L}$ respectively. Then their results are decomposed in column-wise and down sampled by factor 2 . This operation produces four subimages as shown in figure (1).

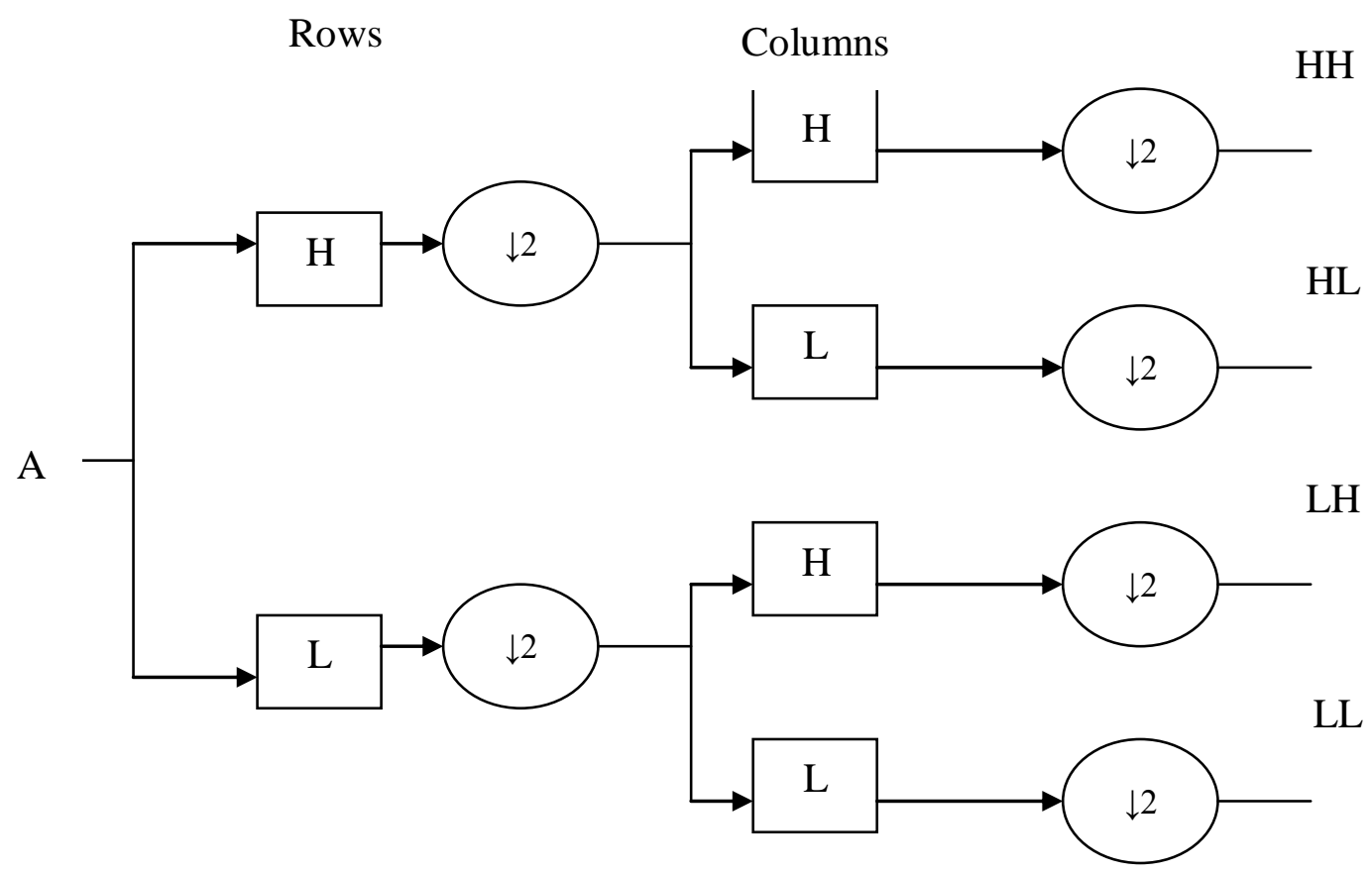

Figure (1): 1-level Wavelet Analysis Filter Bank

The first subimage is represented in the form of low horizontal and vertical frequency denoted by (LL) channel. The second is represented as high horizontal frequency and low vertical frequency denoted by (HL)and known as Horizontal packet. The third is (LH) which represents the low horizontal frequency and high vertical frequency channel known as Vertical Packet. Indeed, $(\mathrm{HH})$ contain high frequency in both directions. These details are shown in figures (2-a) and (2-b) with wavelets decomposition [6] [3]. 


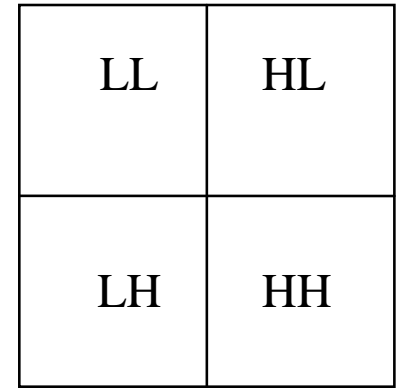

(a)

(b)

Figure (2): Wavelets Frequency Decomposition

Various Literature works used the wavelets coefficient due to their efficiency in face recognition. Related works apply the 3-level subband wavelets transform for human face recognition through Principles Component Analysis (PCA). These subbands decompose an image into different frequency components [6]. The study by Dai \& Yuen (2006) use wavelet to enhance discriminate analysis which is used effectively to solve small region problem such as image face recognition. Also, Mallat S. (1999) work use, 2D discrete wavelet transform which is known as multiresolution face recognition system. The multiresolution extract wavelet coefficients for both spatial and frequency domain [4].

These subimages are known as wavelets-packet, they are linear combinations of wavelets function they reflect the powerful of wavelet bases in representing smooth images. The wavelet packet method is generalization of wavelet decomposition that offers a richer range of possibilities for signal analysis. Also used to construct various types of images with different spatial-frequency structure as shown in figure (3) The wavelets packets have been used in face recognition or face feature extraction [6].
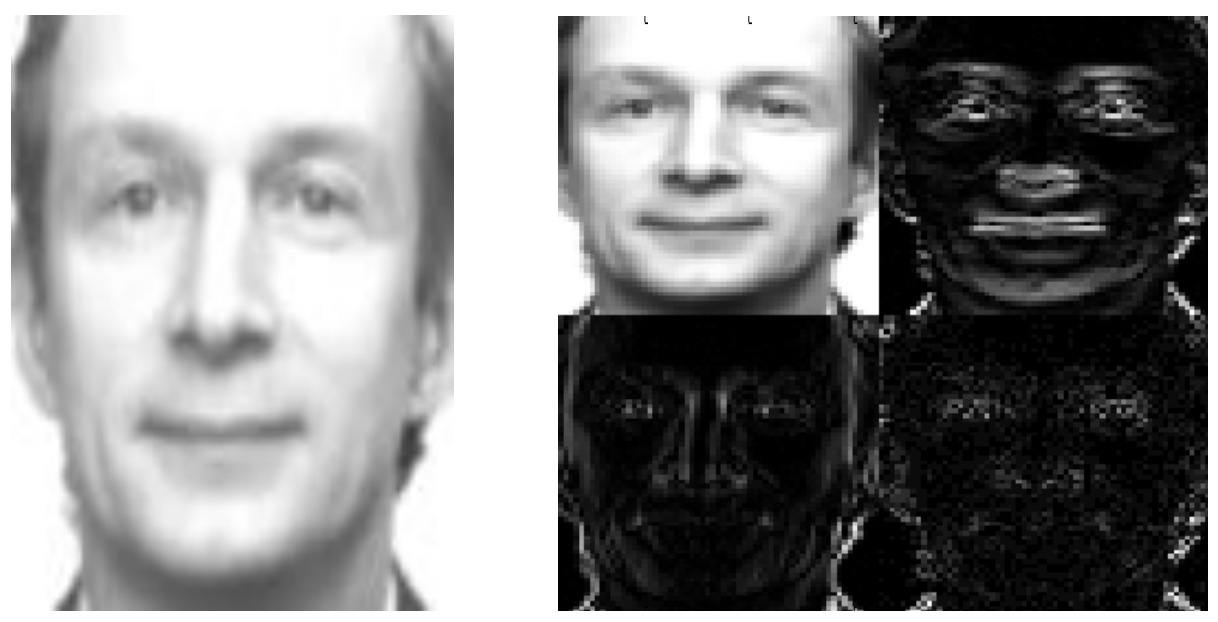

Figure (3): two-dimensional wavelet 1-level decomposition of facial image 


\section{2-1 Wavelet coefficients in Face Recognition}

The wavelet transform or the wavelet packet transform have been used for feature extraction in face recognition. These are used in three ways:

1) The direct use of wavelet coefficients. This method regards the frequency components as features and stored in feature vectors. In general low frequency components produce best comparison power and invariant to image variations. The inefficiency of this method is that it produces irrelevant features due to the irrelevant/redundant information produced by this method [4][6].

2) The combination of wavelets coefficients produced features with minimum loss of information. This method is merged with traditional transformation such as (LDA, PCA) to enhance the discriminative features. Also, the use of different spatial-frequency subbands wavelets coefficients through statistical quantum [6][11].

3) Searches for the best features in the wavelet packet library. This method offered the best discriminative coefficient to represent features in face recognition as a generalization of the wavelet transform, the wavelets packet not only offers us an attractive tool for reducing the dimensionality by feature extraction, but also allows us to create localized subbands of the data in both space and frequency domains [6].

\section{3- The proposed work}

The system use the appearance-based approaches with FERET (Face Recognition Technology), grayscale images of human heads with views ranging from frontal to left and right profile [13]. Also the database is designed with variable factors such as different expressions, different eyewear/hairstyles, with sad, happy, normal, with/ without glass and different illuminations. As well as, different position (near and far) from camera that varied the face view. We construct the visual database image by randomly choose 117 images accompanied with different coordinate as a visual database for training and testing phases. A sample of these images is shown in figure (4).

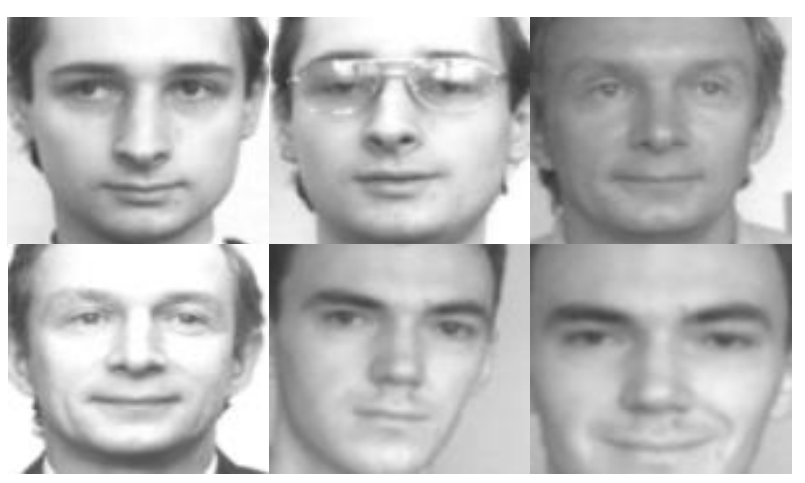

Figure (4): a sample from database images 
Subsequently, each face image is downsampled by bilinear methods to a size of $128 \times 128$. Then these images were forced on fixed face masks then processed. The training set was set up by a random selection of (or) samples per person from the whole database and the testing set was the remaining images. We used a total of 117 images for training and 50 images were selected randomly for testing from this database. Then apply the 2-D 2-level wavelet decomposition for our experiment. We select 2-D HL, LH subimages with $24 \times 24$ pixels each to perform our experiments. These subimages are selected for the reason that, these two subbands contain low frequency components. Generally, they are less sensitive to image variations. As well as these components form the most informative subimage equipped with the highest discriminating power. Then the images are segmented to $6 \times 6$ blocks with 4 pixels per block the block-wise scheme is faster and efficient in processed than pixel-wise scheme. The procedure of the propsed work is shown in figure (5).

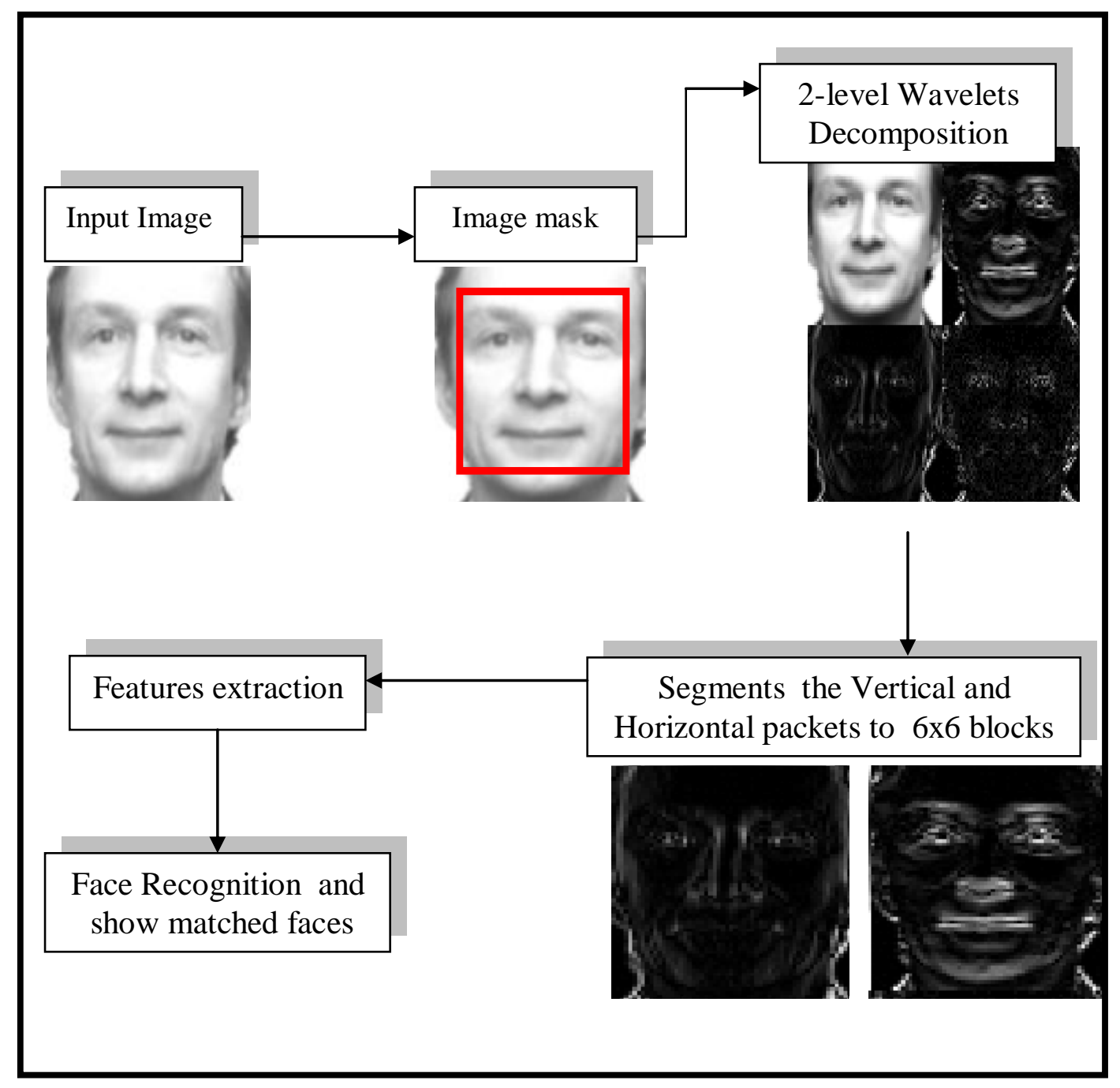

Figure (5): the procedure of the proposed work 


\section{4- Face Feature Extraction}

Features are extracted efficiently through the combination of direct use of Wavelets components and the statistical methods. The statistical moment (second order) is used to compute the standard deviation (STD). The STD shows the contrast of an image. Standard deviation is defined mathematically by equations (2) [2]:

$$
\sigma i j=\left(\frac{1}{N} \sum_{i=j=1}^{N}\left(P_{i j}-\mu_{i}\right)^{2}\right)^{1 / 2}
$$

As shown $\mathrm{P}_{\mathrm{ij}}$ is the value of the $\mathrm{i}$-th gray color components of the image pixel $\mathrm{j}, N$ is the number of pixels in the block, while $\mu_{\mathrm{ii}}$ is the mean of the block ij . It is computed in equation (3) [2]:

$$
\mu_{i j}=\frac{1}{N} \sum_{i=j=1}^{N} P i j
$$

This moment is computed per block and produces feature vector for further process to both query face and the whole dataset images. Each vector represents the horizontal and vertical subimages. These vectors are denoted as STV1Q and STH2Q for query while, STV1 and STH2 for the dataset image as shown in equation (4), (5), (6) and (7) respectively.

$\mathrm{STV} 1 \mathrm{Q}=\left[\sigma_{00}, \ldots \ldots \ldots, \sigma_{23}\right]$

STH2Q $=\left[\sigma_{00}, \ldots \ldots \ldots, \sigma_{23}\right]$

STV $1=\left[\sigma_{00}, \ldots \ldots \ldots, \sigma_{23}\right]$

$\mathrm{STH} 2=\left[\sigma_{00}, \ldots \ldots \ldots, \sigma_{23}\right]$

Then a comparison between the query image to each of the stored images in the database is computed, and the label of the best match is returned. The computation process is done by finding the difference between the query features vector and the data set image vector. This difference is produced by IMSUBTRACT (Matlab function) between (STV1Q and STV1) as well as (STH2Q and STH2). Then, the ten smallest differences with the corresponding image names are stored in new vectors for both horizontal and vertical packets. These vectors are union and the repeated image names are regarded as the most similar face images to be displayed as a recognition results.

\section{5- experimental results}

The work aim to recognize and analyze the faces images. These are done by selecting 117 face images for training and testing from FERET. A sample of the results from the proposed work is shown in table (1). These results are described as values represent the number of relevant database images, number of retrieved images and the number of relevant retrieved images. The results are shown as images in figure (6-b) and (7-b) respond to the entered images in figure (6-a) and (7-a) respectively. 
Table (1): the results of testing the proposed work

\begin{tabular}{|c|c|c|c|}
\hline $\begin{array}{l}\text { Image } \\
\text { Name }\end{array}$ & $\begin{array}{l}\text { NO. of Relevant } \\
\text { Dbase Images }\end{array}$ & $\begin{array}{l}\text { Retrieved } \\
\text { Images }\end{array}$ & $\begin{array}{c}\text { Relevant } \\
\text { Images }\end{array}$ \\
\hline 1 & 10 & 6 & 6 \\
\hline 2 & 7 & 7 & 7 \\
\hline 3 & 10 & 10 & 10 \\
\hline 6 & 6 & 6 & 6 \\
\hline 7 & 10 & 10 & 10 \\
\hline 8 & 10 & 8 & 8 \\
\hline 12 & 4 & 4 & 3 \\
\hline 20 & 5 & 5 & 4 \\
\hline 21 & 4 & 6 & 4 \\
\hline 30 & 6 & 5 & 4 \\
\hline 31 & 10 & 5 & 5 \\
\hline 42 & 4 & 8 & 4 \\
\hline 43 & 9 & 9 & 8 \\
\hline 44 & 11 & 9 & 9 \\
\hline 46 & 9 & 9 & 7 \\
\hline 47 & 9 & 10 & 8 \\
\hline 52 & 5 & 5 & 4 \\
\hline 55 & 5 & 5 & 4 \\
\hline 57 & 6 & 7 & 6 \\
\hline 58 & 6 & 9 & 6 \\
\hline 60 & 6 & 6 & 5 \\
\hline 70 & 8 & 6 & 5 \\
\hline 71 & 8 & 9 & 7 \\
\hline 72 & 8 & 6 & 5 \\
\hline 80 & 3 & 2 & 2 \\
\hline 82 & 8 & 7 & 6 \\
\hline 90 & 10 & 7 & 6 \\
\hline 97 & 10 & 6 & 5 \\
\hline 103 & 10 & 7 & 7 \\
\hline 107 & $\varepsilon$ & 2 & 2 \\
\hline 110 & 6 & 4 & 4 \\
\hline 111 & 6 & 6 & 6 \\
\hline 115 & 6 & 6 & 5 \\
\hline
\end{tabular}




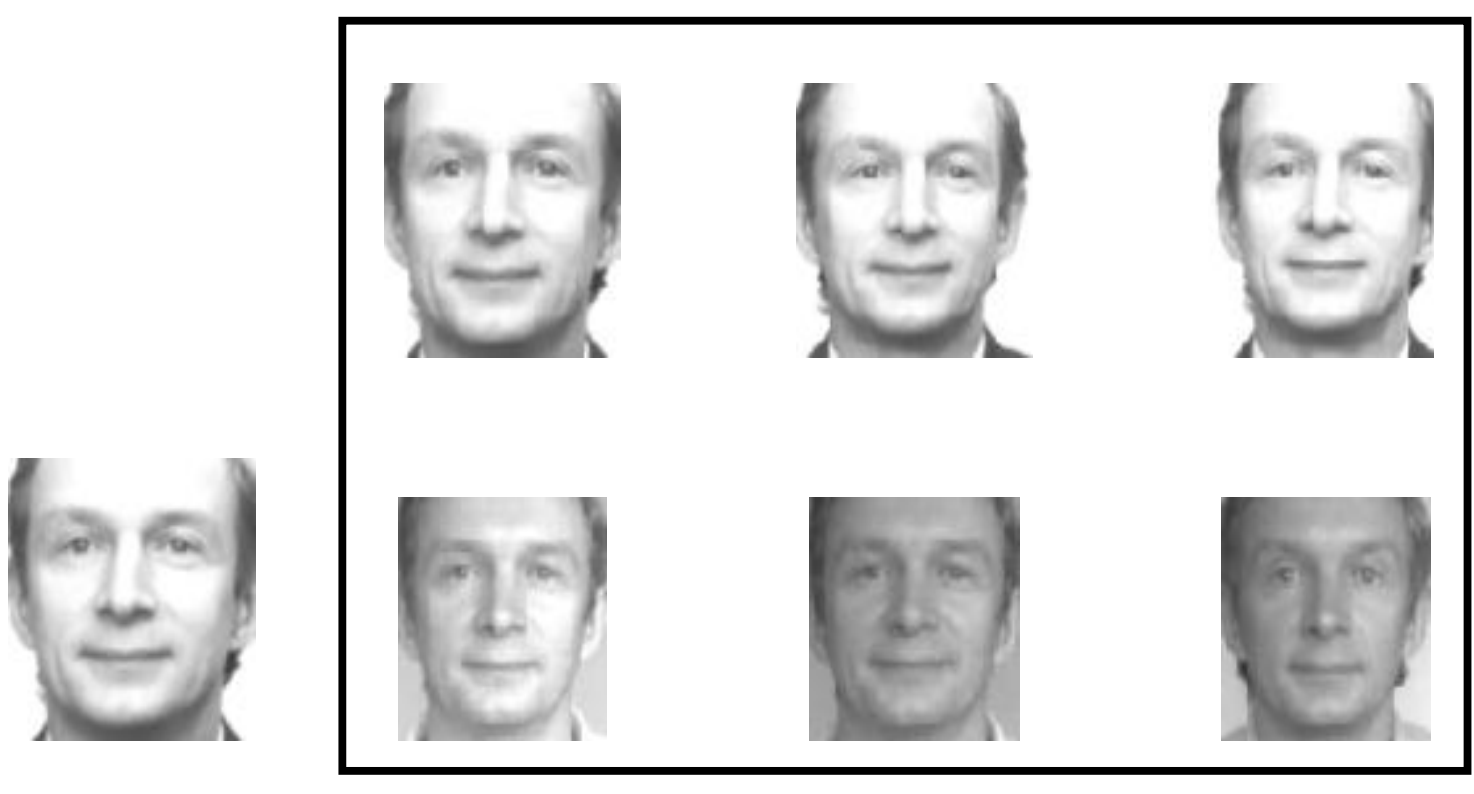

(a)

(b)

Figure (6): the results of the proposed system (a): The query image (b): The Retrieved Images

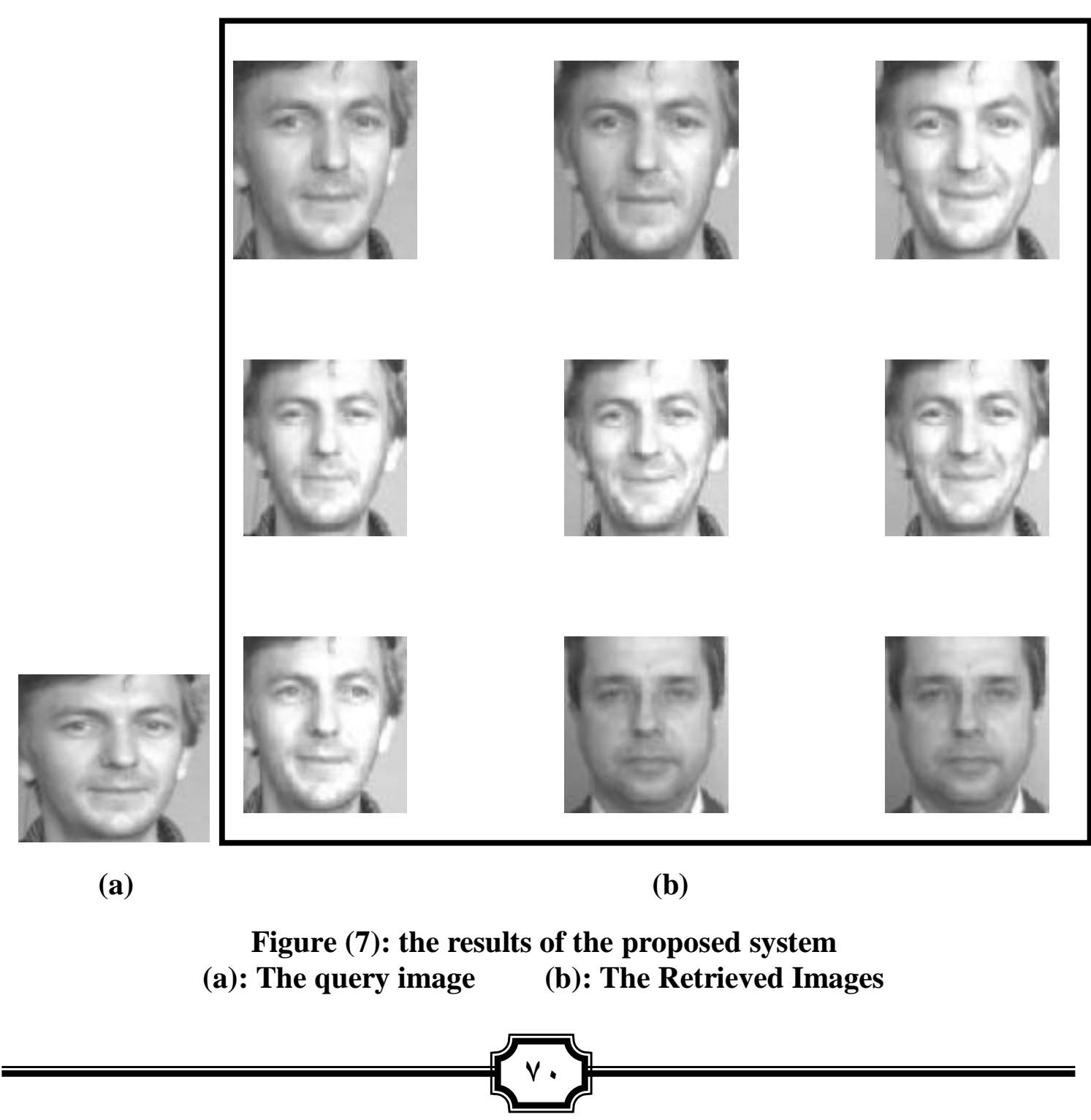




\section{6- Results Analysis}

In order to measure the performance evaluation of the proposed work. The precision and recall are measured over the entire database. The precision measures the ratio between the relevant images retrieved to the total number of images retrieved, the relevant images are recognized manually. Recall measures the percentage of the total, relevant images retrieved. Mathematically Precision $(\mathrm{P})$ and Recall $(\mathrm{R})$ are defined by equations (8) and (9) respectively [9]:

$\mathrm{P}=\frac{N_{R}^{R}}{N_{R}}$

$\mathrm{R}=\frac{N_{R}^{R}}{N}$

Where $\mathrm{N}_{R}^{R}$ is the number of relevant images retrieved, $\mathrm{N}_{\mathrm{R}}$ is the total number of images retrieved and $\mathrm{N}^{\mathrm{R}}$ is the total number of relevant images in the database. The precision and recall of 34 images is selected randomly as a sample from the 50 test sample images and recorded in table 2 .

Table 2: Performance evaluation of the proposed system

\begin{tabular}{|c|c|c|}
\hline Image Name & Precision & Recall \\
\hline 1 & 1 & 0.6 \\
\hline 2 & 1 & 1 \\
\hline 3 & 1 & 1 \\
\hline 6 & 1 & 1 \\
\hline 7 & 1 & 1 \\
\hline 8 & 1 & 0.8 \\
\hline 12 & 0.75 & 1 \\
\hline 20 & 0.8 & 0.8 \\
\hline 21 & 0.67 & 1 \\
\hline 30 & 0.8 & 0.67 \\
\hline 31 & 1 & 0.5 \\
\hline 42 & 0.5 & 1 \\
\hline 43 & 0.89 & 0.89 \\
\hline 44 & 0.82 & 1 \\
\hline 46 & 0.79 & 1 \\
\hline 47 & 0.8 & 0.89 \\
\hline
\end{tabular}


Biometrics Identification based Face Image Authentication.

\begin{tabular}{|c|c|c|}
\hline File Name & Precision & Recall \\
\hline 52 & 0.8 & 0.8 \\
\hline 55 & 0.8 & 1 \\
\hline 57 & 0.86 & 1 \\
\hline 58 & 0.67 & 1 \\
\hline 60 & 0.85 & 1 \\
\hline 70 & 0.84 & 0.62 \\
\hline 71 & 0.78 & 0.88 \\
\hline 72 & 0.83 & 0.62 \\
\hline 80 & 0.67 & 1 \\
\hline 82 & 0.75 & 0.85 \\
\hline 90 & 0.86 & 0.6 \\
\hline 82 & 0.86 & 0.75 \\
\hline 97 & 0.84 & 0.5 \\
\hline 103 & 1 & 0.7 \\
\hline 107 & 1 & 0.5 \\
\hline 110 & 1 & 0.67 \\
\hline 111 & 1 & 1 \\
\hline 115 & 0.84 & 1 \\
\hline
\end{tabular}

The performance evaluation of our proposed system is plotted in figure (8). This figure shows that most of precision and recall values are ranged between 0.7 up to 1 which reflects the enhancement of this work.

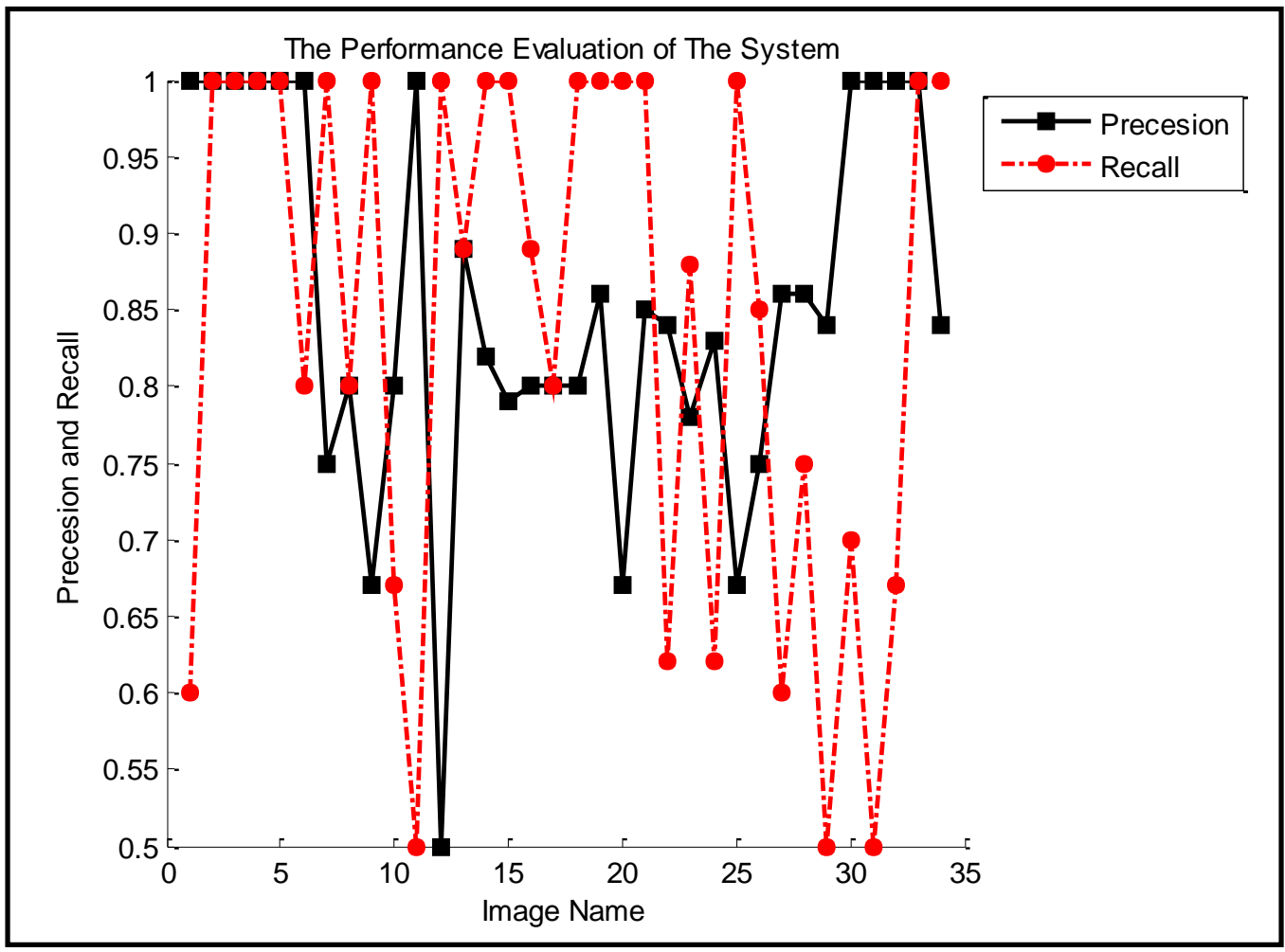

Figure (8): the performance evaluation of the proposed system 
In order to test the efficiency of the proposed work, we compare our proposed work presented above with another related work used face images from FERET visual database. That work is titled "Face Recognition by Applying Wavelet Subband Representation and Kernel Associative Memory" [12]. The recognition accuracies (precision) of the related work vary from $83.3 \%$ to $91.6 \%$. The recognition accuracies (precision) of the related work vary from $75 \%$ to $100 \%$. These results show the enhancement of the proposed work over the related work, where the accuracies exceeds the measures of the others work. Table (3) shows the results of the comparison.

Table (3): Performance Evaluation Comparison

\begin{tabular}{|c|c|c|c|c|}
\hline Value & \multicolumn{2}{|c|}{ Proposed work } & \multicolumn{2}{c|}{ Related work } \\
\hline $\begin{array}{c}\text { Recognition Rate } \\
\text { Percentage }\end{array}$ & Minimum & Maximum & Minimum & Maximum \\
\hline
\end{tabular}

\section{- Conclusion}

Here, proposing for a bioinformatics system through the face recognition. The difference between the proposed work and others is that, we select the 2D wavelets vertical and the horizontal subimages. While others use the whole four subimages or isolate ever face region from approximation packet. The selected images were segmented to blocks. The image is treated in block-wise scheme while the others use pixelwise scheme. The block-wise is performed faster than others method that used pixel-wise scheme. Successful results were achieved for automated face recognition system. These results were measured with Precision mostly varied between $70 \%$ to more than $90 \%$ and Recall ranged from $80 \%$ to $100 \%$.

In future work we try to use colored face images. Also this system will be developed to be used for security system area. Finally an idea to develop the system to detect and isolate face image from combined images.

\section{References}

[1] B. J. Axnick Karl and C. Ng. Kim, (2005), "Fast Face Recognition", Proceeding of Image and Vision Computing Newzland Conference, Newzland.

[2] C. Gonzalez Rafael and E. Richard Woods, (2002), "Digital Image Processing", $2^{\text {nd }}$ Ed., Prentice-Hall, New Jersey, USA, P:793. 
[3] Chen Junqing, (2003), Perceptually-based texture and color features for image segmentation and retrieval, A Ph.D. Thesis Submitted to Northwestern University, Illinois, USA.

[4] Dai Dao-Qing and Yan Hong, (2007), "Wavelets and Face Recognition", the Journal of Machine Learning Research, Vol. 8, P:1165-1195.

[5] Ekenel Kemal Hazim and Stiefelhagen Rainer (2006), "Analysis of Local Appearance-Based Face Recognition: Effects of Feature Selection and Feature Normalization", Proceeding of Computer Vision and Pattern Recognition Conference (CVPR06).

[6] Fladsrud Tom, (2005), "face recognition in a border control environment", MSc Thesis submitted to the Department of Computer Science and Media Technology, Gjøvik University College, 2005

[7] Gunduz Aysegul, (2003), "Compression and Transmission of Facial Images over Very Narrowband Wireless Channels", MSc. thesis submitted to the Graduate Faculty of North Carolina State University, Electrical and Computer Engineering, USA.

[8] Kepenekci Burcu, (2001), "Face Recognition using Gabor Wavelet Transform", MSc Thesis Submitted To The Graduate School of Natural Sciences of The Middle East Technical University, Dept. of Electrical and Electronics Engineering.

[9] Orbel Sabri Bough, Tarel Jean Philippe and Boujemaa Nozha, (2005). "Generalized histogram intersection kernal for image recognition", Proceeding of International Conference on Image Processing, Genoa, Italy: 161-164.

[10] S. Feris Jim Rogerio, Kentaro Toyama Gemmell and Kr"uger Volker, (2002), "Hierarchical Wavelet Networks for Facial Feature Localization", Proceeding of the Fifth IEEE International Conference on Automatic Face and Gesture Recognition.

[11] Sanderson Conrad and K. Paliwal Kuldip, (2003), "Fast Features for Face Authentication under Illumination Direction Changes", Pattern Recognition Letters 24: 2409-2419.

[12] Zhang Bai-Ling, Zhang Haihong, and Ge Sam Shuzhi, (2004), "Face Recognition by Applying Wavelet Subband Representation and Kernel Associative Memory", Proceeding of IEEE Transactions on Neural Networks, vol. 15(1).

[13] FERET Image Database http://www.nist.gov/humanid/feret/feret _master.html [Internet]. 